\section{Cómo responder a una epidemia de dengue: visión global y experiencia en Puerto Rico}

\author{
José G. Rigau-Pérez 1 \\ y Gary G. Clark ${ }^{1}$
}

Palabras clave: dengue, brotes de enfermedades, vigilancia epidemiológica.

\footnotetext{
Dengue Branch, Division of Vector-Borne Infectious Diseases, National Center for Infectious Diseases, Centers for Disease Control and Prevention, San Juan, Puerto Rico 00920-3860. La correspondencia debe dirigirse a Gary G. Clark, CDC Dengue Branch, 1324 Calle Cañada, San Juan, Puerto Rico 00920-3860. Correo electrónico: ggc1@ cdc.gov
}

El dengue es una enfermedad viral endémica y frecuentemente epidémica en muchos países tropicales. Aunque su incidencia en el mundo está en continuo aumento, a escala local se observan ciclos de alta y baja actividad de duración variable, lo cual hace que sea difícil predecir las epidemias, es decir, las situaciones en que la incidencia es mayor de la esperada o aumenta fuera de época, en comparación con otros años.

En las zonas endémicas de dengue se producen otros fenómenos potencialmente devastadores comparables con las epidemias, como los huracanes, que se presentan de forma recurrente pero errática. A pesar de que los huracanes no se pueden controlar ni eliminar, muchas sociedades han aprendido a tomar medidas para reducir las pérdidas de vida y de propiedades que ocasionan estos fenómenos meteorológicos. Sin embargo, todavía se ven con frecuencia señales de que las epidemias no se enfrentan de manera óptima. Entre esas señales se encuentran los mensajes de alerta tardíos que llegan a la población cuando la epidemia se aproxima a su pico máximo (lo que indica que hay problemas en el sistema de vigilancia o en el establecimiento del plan de contingencia), las cifras de mortalidad superiores a $1 \%$ de los casos de dengue hemorrágico (lo que indica que hay problemas en el sistema de atención médica) y las especulaciones acerca de que la epidemia se debe a algún virus o mosquito mutantes, a otra causa emergente o a varias enfermedades concurrentes (lo que indica que no se han reconocido los problemas de atención médica y que se busca la explicación en hipótesis científicas más que en deficiencias reales) (1-3).

La forma ideal de controlar una enfermedad es la prevención primaria, es decir, evitar que las personas enfermen. Esto, en el caso del dengue, significa interrumpir la transmisión mediante la inmunización de las personas o la eliminación de los mosquitos. Sin embargo, todavía no se cuenta con una vacuna contra esta enfermedad y los métodos actuales para el control de los vectores más frecuentes - los mosquitos Aedes aegypti y Aedes albopictusson poco eficaces (4). Tampoco se conoce ninguna estrategia que haya logrado frenar una epidemia de dengue.

Estas epidemias producen crisis de larga duración (siete meses en promedio). Por consiguiente, los esfuerzos gubernamentales deben sostenerse durante todo ese período (5). En una epidemia de dengue, las agencias de salud pública deben refor- 
zar la prevención secundaria, garantizar el reconocimiento temprano de la situación y el tratamiento apropiado de los enfermos, evitar que las epidemias menoscaben el funcionamiento de las clínicas, hospitales e industrias, y conseguir datos fiables y útiles para ayudar a las autoridades de salud pública y a la población a tomar medidas para evitar la enfermedad.

Hace más de 20 años, Gubler desarrolló en Puerto Rico un programa de vigilancia y control de dengue con elementos que todavía se recomiendan en el marco de referencia de la nueva generación de programas de prevención y control del dengue en las Américas promovido por la Organización Panamericana de la Salud (OPS) y en la estrategia para el control del dengue elaborada más recientemente por la Organización Mundial de la Salud (OMS) (6-9).

La experiencia acumulada en Puerto Rico y en otros países permite reconocer algunos componentes de los programas de control que han sido eficaces para reducir el impacto de estas epidemias. El presente artículo describe las áreas de atención prioritaria en que se debe trabajar para reducir el impacto de las epidemias de dengue, y que también pueden adaptarse para combatir otros tipos de epidemia.

\section{ÁREAS DE ATENCIÓN PRIORITARIA}

\section{Comité de acción intersectorial y plan de acción de emergencia}

La infestación con mosquitos depende no solo del clima, sino también del conocimiento y de la actitud de la población, de la eficacia y cobertura de los programas gubernamentales de control del vector, del adecuado abastecimiento de agua, y del reciclaje o eliminación apropiada de los residuos sólidos. La probabilidad de que se transmita la infección es resultado de varios factores, entre ellos el grado de urbanización del entorno, la densidad de la población y la frecuencia con que se trasladan las personas desde zonas del mundo donde circulan diferentes serotipos del virus (6). Por su parte, la capacidad de controlar una epidemia depende de la facilidad con que la población puede acceder a los servicios médicos y de diagnóstico, del historial de transparencia de la acción del gobierno y de la confianza de la población en las estadísticas que se le presentan. Por lo tanto, el desarrollo de casos graves depende a la vez de factores intrínsecos del paciente y de factores históricos y sociales.

No es posible que una sola agencia gubernamental pueda controlar todas las causas y consecuencias de una epidemia y proteger la salud de la población. Es por ello que las oficinas regionales de la OMS recomiendan a los países en los que el dengue es endémico que establezcan acciones intersectoriales para combatir la enfermedad (10-12). Las guías publicadas por la OPS en 1994 recomiendan nombrar un coordinador nacional, mientras que el marco de referencia de la nueva generación de programas indica explícitamente que estos coordinadores deben tener "apoyo político y financiero firme con coordinación intersectorial eficaz" y "actuación intersectorial coordinada a todos los niveles" (13). Esto exige la labor de más de una persona. Aunque la guía para la planificación de operaciones de emergencia por riesgos de cualquier tipo elaborada por la Agencia Federal para el Control de Emergencias, de los Estados Unidos de América, no cubre situaciones de epidemia, presenta un esquema muy útil y recomienda la creación de un equipo que represente a las organizaciones vinculadas con el esfuerzo de respuesta a una emergencia (14). Para la coordinación intersectorial, en ese comité deben participar no solo miembros de las agencias gubernamentales (como las encargadas de la salud, la agricultura, la educación, el orden interior, la hacienda pública y los servicios de agua potable y de eliminación de desperdicios), sino también de diversas entidades privadas (filantrópicas, industriales, comerciales, de educación privada, uniones laborales y sindicatos). Este comité debe actuar contra el dengue aun cuando no haya epidemias y debe preparar un plan de acción de emergencia en el que se plasme el más firme compromiso de poner en práctica con rapidez las medidas necesarias previstas en caso de epidemia. En el cuadro 1 se resumen las recomendaciones aplicables a epidemias locales, regionales o nacionales, organizadas según el momento en que se debe tomar una decisión o emprender una acción.

\section{Reconocimiento precoz de la epidemia}

De la misma forma en que los meteorólogos anuncian una depresión tropical antes de que se convierta en una tormenta y finalmente en un huracán mediante la vigilancia y la interpretación de señales de alerta, los epidemiólogos pueden identificar indicios precoces del aumento de la actividad del dengue (15). En zonas sin vigilancia de laboratorio o antes de que un caso de dengue típico provoque el envío de muestras para su diagnóstico, la señal inicial del aumento en la actividad de dengue puede estar dada por el incremento de los casos febriles en las clínicas ambulatorias o de las ventas de antipiréticos en las farmacias (16).

En Puerto Rico, donde no se utiliza como indicador el aumento de las poblaciones de mos- 


\section{CUADRO 1. Áreas de atención prioritaria para prever y responder a una epidemia de dengue, agrupadas según el momento} de acción

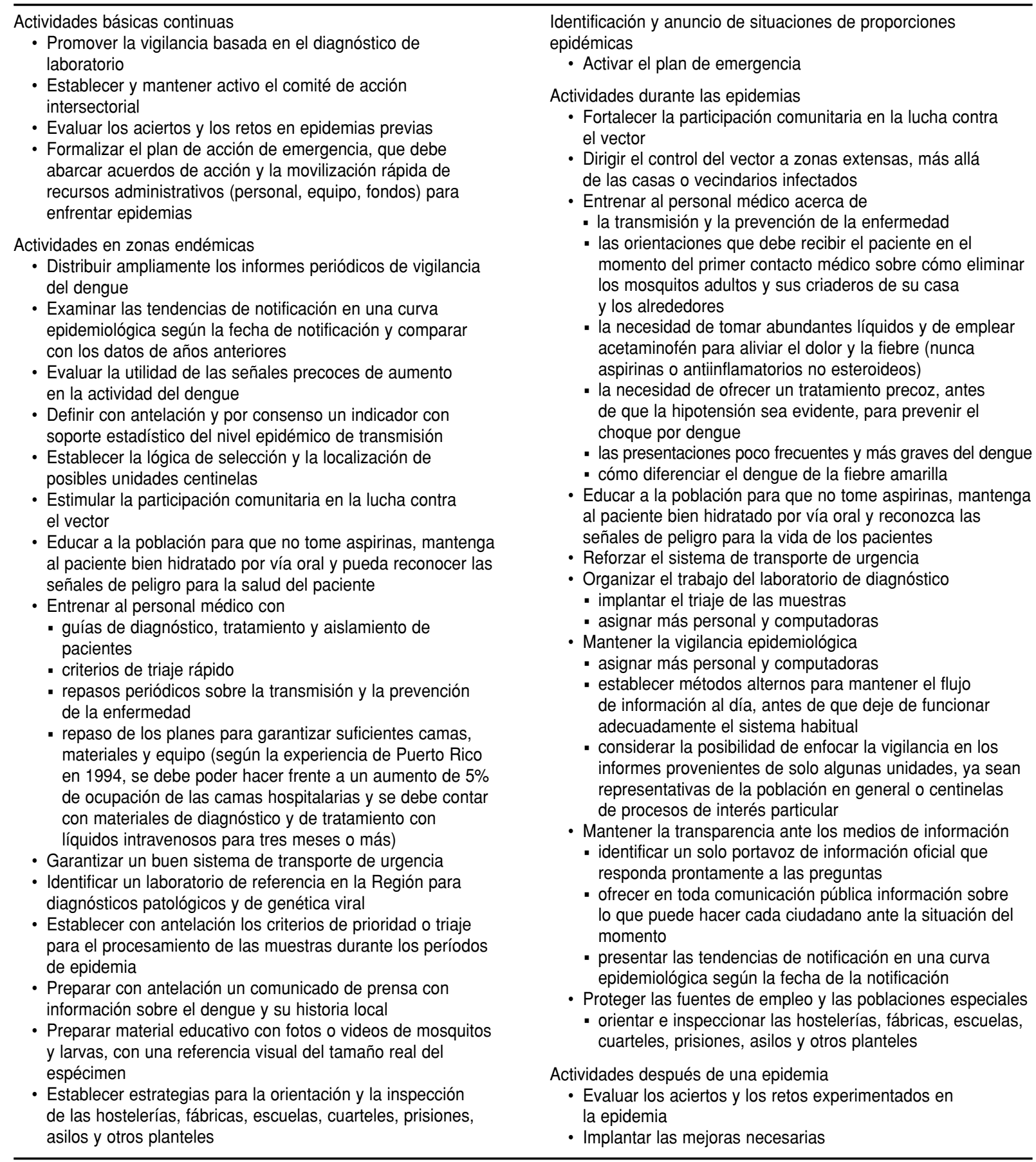

quitos, sino los niveles de notificación de la enfermedad y los resultados de los laboratorios de diagnóstico, la señal más temprana de una epidemia general de dengue es el aumento en la tasa de positividad de las pruebas de aislamiento de virus en los casos recibidos en el mes de mayo (17). En la curva de incidencia, el número de casos durante los meses de abril a junio es generalmente el más bajo. Si la tasa de positividad de los sueros obtenidos en los primeros cinco días de enfermedad sometidos a pruebas de aislamiento de virus (por cultivo celular o inoculación de mosquitos) es mayor de $10 \%$ para 
mayo, hay una alta probabilidad de que ocurra una epidemia en una o varias regiones o en todo el país antes de finalizar el año. Esto fue así en cuatro ocasiones entre 1988 y 2003: dos veces antes de epidemias nacionales (1994 y 1998) y dos veces antes de epidemias en alguna región del país (2001 y 2003). $\mathrm{Si}$ se toma como criterio de epidemia nacional el aumento de la incidencia a más de 30 casos por 10000 habitantes, tres años cumplen ese criterio: los ya mencionados 1994 y 1998 y el año 1992 (tasa de 31,5 por 10000 ), en el que se observó un nivel de notificación moderado, pero durante casi todo el año). La señal de alerta temprana se manifestó solo en 1994 y 1998 (66,6\% de sensibilidad) y se hubiera establecido la alarma en dos ocasiones durante los 13 años en que no se presentaron epidemias (85\% de especificidad).

La elevación de la tasa de positividad al virus en las pruebas de laboratorio en mayo permite prever con 4-8 semanas de antelación el despegue de una epidemia de dengue (que generalmente ocurre entre junio y agosto), pero el inicio temprano de la temporada de dengue - es decir, el incremento de al menos unos pocos casos antes del momento en que generalmente se eleva la curva endémica- es también una señal, aunque más tardía. La notificación de casos rebasó el nivel endémico cuatro semanas antes del aumento geométrico en la epidemia de 1998.

En Puerto Rico se elabora una curva semanal de los posibles casos de dengue según la fecha de recibo de la notificación (ver más adelante) y se compara con el promedio esperado de casos y su canal endémico (promedio \pm 2 desviaciones estándar). También se ha establecido una señal de alerta basada en las notificaciones de casos de cada municipio en relación con el valor esperado (18) (figura 1, segunda gráfica). Estas dos gráficas basadas en los casos no confirmados constituyen ejemplos de vigilancia sindrómica, ya que solo se toma en cuenta el cuadro clínico (síndrome viral febril con algunos de los síntomas de dengue) (19). Como el objetivo de la vigilancia en Puerto Rico es la prevención, estas gráficas forman parte de los informes semanales que se envían a las autoridades sanitarias, hospitales, programas de educación de posgrado en salud pública y a otras instituciones interesadas (figura 1), y están disponibles en la página de Internet del Departamento de Salud de Puerto Rico (http:/ / www.salud.gov.pr).

\section{Alerta en torno a las epidemias}

El epidemiólogo a cargo del sistema de vigilancia de dengue debe conocer en detalle cómo funciona ese sistema y, en particular, los factores hu- manos que lo afectan. Debe luchar contra las fuerzas burocráticas que lo mantienen sujeto a su escritorio (como describe Kafka tan agudamente en su relato Poseidón) y visitar con frecuencia los hospitales, laboratorios, oficinas locales y regionales donde se realizan la notificación y el registro de los casos (20). Es opinión tan generalizada como errónea que el epidemiólogo se dedica al análisis de "los números". Por el contrario, gran parte del esfuerzo del epidemiólogo (sobre todo si está dedicado a la práctica en instituciones gubernamentales) se dirige a promover la acción al explicar, crear consenso, advertir y señalar errores y evaluar resultados.

El reconocimiento de una epidemia es, en última instancia, un acto humano y el proceso mediante el cual las personas enfrentan las malas noticias se conoce como la reacción de duelo. Le corresponde al epidemiólogo, como parte de sus destrezas profesionales, pasar rápidamente las etapas de ese proceso y preparar a sus colegas y superiores para la respuesta, según reaccionen sucesivamente con negación, ira, depresión, negociación y finalmente aceptación (21). El diagnóstico de una epidemia se presta mucho a la tergiversación (reacción de duelo multiplicada por el número de personas en la cadena de mando), por lo que el mensaje debe ser explicado directamente por quien lo origina. Los profesionales que diagnostican la epidemia (por lo general epidemiólogos y virólogos) deben informar a sus superiores según la línea de comunicación establecida, pero los dirigentes políticos deben oír directamente de ellos (en presencia de sus superiores) la información y sus recomendaciones. Para facilitar este proceso se recomienda que el grupo multidisciplinario que dirige la lucha contra las epidemias de dengue (clínicos, entomólogos, administradores y políticos, además de los epidemiólogos) reciba sistemáticamente los informes periódicos de vigilancia del dengue previo a las epidemias.

Es muy importante que se hayan acordado con antelación los criterios del nivel epidémico de transmisión, para lo cual se deben utilizar indicadores fundamentados en las estadísticas $(22,23)$. De lo contrario, el estado emocional de negación buscará refugio en las diferencias entre los términos "brote" y "epidemia", que en epidemiología son la misma cosa: situaciones (períodos) en las cuales los casos aparecen con mayor frecuencia de la esperada para una población y localidad específicas.

Aunque la incidencia nacional sea baja, se debe tener en cuenta que esta es el resultado de la integración de los datos de muchas áreas con poca o ninguna transmisión y de epidemias en pequeñas poblaciones que no deben quedar desatendidas. Además, se debe tomar en consideración que hay períodos en los que los niveles de transmisión son moderados, pero tan prolongados o generalizados 
FIGURA 1. Informe de vigilancia del dengue en Puerto Rico con base estadística, del 29 de noviembre al 5 de diciembre de 1998

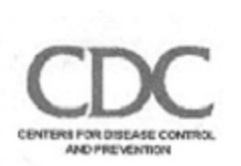

Número de casos notificados por semana de recibo, 1997-1998

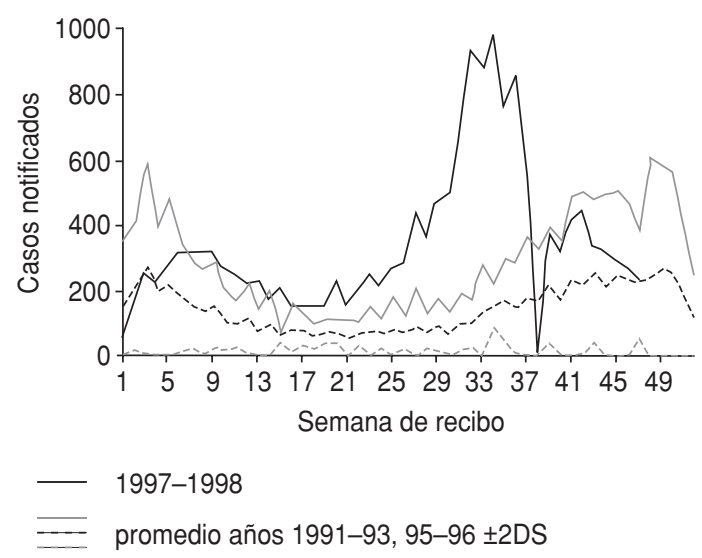

A la semana número 47 de 1998 el número de casos notificados se encuentra al mismo nivel que el promedio para los últimos cinco años.

Positividad en la prueba de detección de anticuerpos lgM contra dengue efectuada en la semana 48

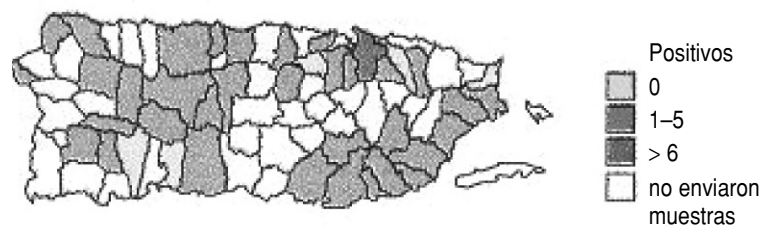

Fechas de comienzo de síntomas 09/20/98-11/26/98 mediana 11/12/98

Las muestras procesadas para IgM contra dengue en la presente semana indican transmisión de dengue en 36 pueblos con una mayor actividad de la enfermedad en San Juan.

que el total de casos puede ser mayor que el observado durante una epidemia de gran envergadura.

\section{Trabajo de los laboratorios de diagnóstico}

Durante una epidemia, los laboratorios de diagnóstico literalmente se inundan de sangre, debido al elevado número de muestras que reciben. Antes de utilizar pruebas comerciales que permitan obtener resultados rápidos se debe comprobar que
Pueblos con aumento significativo de casos de dengue notificados en las semanas $45-48$

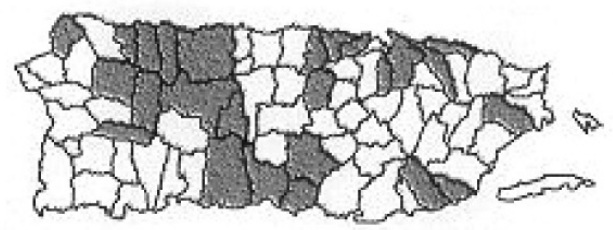

Casos (notificados/esperados) durante este período:

Aguadilla (3/1), Arecibo (14/4), Camuy (10/1), Carolina (31/12), Coamo (8/2), Corozal (8/3), Dorado (6/2), Guaynabo (26/9), Hatillo (5/1), Jayuya (7/1), Juana Díaz (5/1), Lares (7/1), Loíza (6/1), Maricao (4/1), Maunabo (5/1), Naguabo (5/1), Patillas (22/6), Ponce (44/14), Quebradillas (3/1), San Juan (92/52), San Sebastián (8/1), Santa Isabel (3/1), Toa Baja (14/5), Utuado (10/1) y Vega Alta (7/2).

Estos 25 pueblos tienen un aumento significativo en las últimas cuatro semanas en comparación con lo esperado cuando la actividad de dengue es mínima (promedio de cuatro semanas de los meses de abril, mayo y junio, 1989-93).

Positividad en las pruebas de detección de anticuerpos lgM contra dengue efectuadas en las semanas $45-48$

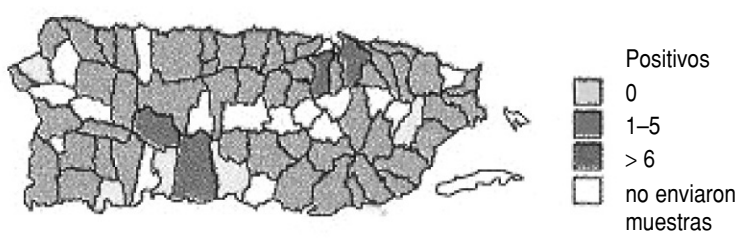

Fecha de comienzo de síntomas 09/20/98-11/26/98 mediana 11/01/98

Las muestras procesadas para lgM contra dengue en las últimas cuatro semanas indican transmisión de dengue en 54 pueblos, con una mayor actividad de la enfermedad en Adjuntas,

Bayamón, Ponce y San Juan.

su sensibilidad y especificidad sean adecuadas. Además, se debe recordar a los médicos y a los pacientes que los resultados negativos obtenidos con las muestras tomadas durante la etapa aguda de la enfermedad no son suficientes para descartar un posible diagnóstico de dengue y deben confirmarse con muestras de suero tomadas durante la etapa de convalecencia.

No es posible ni necesario realizar el diagnóstico de dengue a todos los casos que se solicita. Por otra parte, la atención médica del paciente no 
puede esperar hasta que estén listos los resultados de laboratorio, que pueden tardar días. Por lo tanto, para procesar el elevado volumen de muestras relacionadas con el sistema de vigilancia se deben establecer criterios de prioridad o triaje. Para que este sistema funcione debe haber una comunicación buena y frecuente entre los analistas de laboratorio, los epidemiólogos y los clínicos, de lo contrario ninguno de los tres grupos confiará en la labor ni en los datos que proveen los otros.

En Puerto Rico se establecieron en 1992 los criterios para el procesamiento de las muestras durante períodos de epidemia de dengue. Estos criterios, difundidos ampliamente, exigen una información mínima de cada caso: datos personales del paciente, dirección, fecha de comienzo de los síntomas, gravedad, fecha en que se obtuvo la muestra e institución que notifica el caso. El análisis se realiza según el siguiente orden de prioridad: casos mortales, casos en cuidado intensivo, casos hospitalizados y casos provenientes de municipios donde no se haya detectado aún la transmisión epidémica. Las muestras que no se identifican como prioritarias y que por el volumen de muestras que debe procesar el laboratorio no se analizan en un mes, se eliminan del turno de análisis. Sin embargo, esas muestras deben conservarse organizadas adecuadamente durante varios meses, pues la notificación de un caso mortal (que exige una investigación minuciosa) en ocasiones llega semanas después de la muestra que se tomó durante la evaluación inicial del paciente.

\section{Vigilancia del desarrollo de la epidemia}

Es recomendable examinar las tendencias de la notificación en una curva epidemiológica según la fecha en que la notificación se hizo y comparar los datos con los de años anteriores. La primera reacción es asignar los casos a la fecha en que comenzaron los síntomas, porque esta se deriva directamente de la fisiopatología de la enfermedad y no depende de la tardanza del paciente en ver al médico, del médico en notificar el caso y de la oficina de vigilancia en registrarlo. Sin embargo, es evidente que de esta forma no se puede conocer el número total de casos que comienzan un mismo día hasta mucho después, mientras que la fecha en que se recibe la muestra queda registrada para todos los casos y el sistema de vigilancia puede saber al final de cada día el número de casos notificados. Esto permite comparar el total de ese día con los valores promedio e intervalos de confianza de un canal endémico con respaldo estadístico. Estas características confieren mayor robustez para hacer el segui- miento de una epidemia a la fecha en que se reciben las muestras que a la fecha en que comienzan los síntomas. La curva semanal basada en esta última refleja mejor la situación biológica, pero no se puede elaborar hasta el final del brote.

Por definición, las epidemias consisten en la aparición de un número de casos mayor del esperado. De ahí que los recursos empleados habitualmente para la entrada y el análisis de los datos puedan resultar insuficientes durante las epidemias. La respuesta habitual es buscar más personal y computadoras, pero el atraso en los análisis aumenta mientras se adiestra el nuevo equipo. Por ello, antes de llegar al congestionamiento, es decir, mientras todavía funciona bien el sistema normal, se deben establecer métodos alternativos que permitan mantener el flujo de información al día.

En 1994 y 1998 se estableció en San Juan un sistema paralelo abreviado para la entrada de los datos pertenecientes a los casos cuyas muestras no se procesaban. Sin embargo, esto no fue suficiente para mantener al día el procesamiento de los datos durante la gran epidemia de 1994. Se recurrió entonces a las enfermeras epidemiólogas de los hospitales, que aceptaron contestar cada jueves las llamadas del centro de control e informaban del número de camas ocupadas el día anterior por pacientes ingresados por trombocitopenia o con un diagnóstico presuntivo de dengue o de dengue hemorrágico. Con esta llamada se logró conocer, semana por semana, el curso de la epidemia (17). Que las llamadas se hicieran un día fijo y la parquedad del dato informado (una cifra) simplificaron inesperadamente el sistema, pues las enfermeras epidemiólogas podían dejar la información con una secretaria y las llamadas del centro de control no interferían con sus labores habituales. Incluso, algunas se tomaron la iniciativa de realizar ellas mismas las llamadas para dar la información.

Cuando aumenta la incidencia de la enfermedad salen a la luz los casos de dengue atípicos, así como los de transmisión perinatal y nosocomial (24). Aunque no se ha documentado la transmisión del dengue por transfusión, la magnitud del riesgo durante la reciente epidemia del virus del Nilo occidental que tuvo lugar en los Estados Unidos de América apunta a que esta vía también puede servir para la transmisión de dengue (25).

La vigilancia de laboratorio a gran escala es costosa y, en el caso de síndromes poco frecuentes, puede implicar un gasto enorme por cada caso diagnosticado. Un método alternativo que permite hacer una inversión más eficiente de los recursos es el empleo de unidades centinela o puestos de observación en lugares estratégicos donde se cree que puede presentarse por primera vez el fenómeno clí- 
nico de interés, ya sea la entrada de un virus o los primeros casos de un síndrome (23). La selección de estos lugares se debe basar en el análisis de la información sobre los factores de riesgo para el problema que se vigila. Las unidades centinela exigen recursos, pero permiten hacer un uso más eficiente de ellos. Es necesario realizar un seguimiento personal detallado, frecuente y persuasivo. La información que proviene de los centros centinela es puntual y, por definición, no es representativa de toda la población (5). Sin embargo, puede haber centros centinela no representativos - como las unidades de cuidados intensivos- que revisten un particular interés.

\section{El control de mosquitos}

La respuesta gubernamental a una epidemia de dengue comienza, como es lógico, con la intensificación de los esfuerzos por eliminar el vector. A veces, esta es la principal o única tarea a la que se asignan recursos, lo que impide poner en marcha los múltiples programas que se requieren para controlar la epidemia. Hay guías para dirigir la acción antivectorial en las recomendaciones de la OPS para las Américas (11) y en otras más detalladas aun que han sido emitidas por la oficina regional de la OMS para el sur y el este de Asia $(12,26)$.

Antes de optar por la aplicación de larvicidas o de adulticidas nebulizados en volumen ultra bajo (VUB) se debe evaluar la capacidad del sistema para llevar a cabo el procedimiento con la minuciosidad de cobertura necesaria. La aplicación de insecticida por VUB desde aviones no logra reducir lo suficiente las poblaciones de Ae. aegypti adultos (27). Aunque este procedimiento es útil para combatir otros problemas de salud - como los brotes del virus del oeste del Nilo- para el dengue no solo es ineficaz, sino que produce una injustificada sensación de seguridad en la población y obstaculiza la adopción de prácticas más eficaces, aunque más trabajosas, como la eliminación de criaderos. En algunos lugares, si el número de notificaciones no es muy alto, se dedican los recursos a investigar los domicilios de los enfermos y sus alrededores. Más tarde, según aumentan la intensidad y la extensión geográfica de la epidemia, se reorienta la respuesta a un plan de cobertura general. Esta reacción es, paradójicamente, ineficaz y lenta. Al estudiar la dispersión del dengue en Puerto Rico con sistemas de información geográfica se pudo observar que la enfermedad se difundió abruptamente, ya que el número de vecindarios afectados en un municipio aumentó de dos a seis en solo dos semanas, lo que impidió al sistema de vigilancia de casos definir las rutas (28). Además, por lo general hay un subregistro considerable de los casos de dengue $(29,30)$, así que las actividades de control del vector deben dirigirse a todo el municipio tan pronto se detecte el primer caso. Sin embargo, esta recomendación se deriva de un país con una altísima densidad poblacional, por lo que se recomienda evaluar su aplicación en otros escenarios.

Es importante que las autoridades no culpen a la comunidad de la epidemia, sino que aprovechen la oportunidad para educar a la población y fomentar su colaboración. Se debe recalcar no solo la necesidad de eliminar los criaderos, sino también la importancia de la protección personal contra las picadas del vector mediante el uso de repelentes, ropa protectora y mosquiteros, así como de tela metálica en las puertas y las ventanas de las viviendas. Estos mensajes pueden resultar incomprensibles si no se elaboran de acuerdo con el nivel de comprensión de la ciudadanía. Por ejemplo, $82 \%$ de los miembros de una comunidad de El Salvador encuestados durante la epidemia del año 2000 sabían que los mosquitos trasmiten el dengue, pero solo $33 \%$ habían tomado alguna medida contra posibles criaderos de larvas (31). Esos hallazgos observados durante un brote permiten reenfocar los mensajes educativos para motivar la acción eficaz de los ciudadanos, pero denotan que, aunque la participación comunitaria en la lucha contra el vector es indispensable, las medidas educativas no dan resultados inmediatos y se debe trabajar en ellas con antelación (32).

\section{La protección de las poblaciones especiales y de las fuentes de empleo}

Las epidemias de dengue, por su rápida extensión y larga duración, reducen la capacidad productiva y el desarrollo económico de todos los segmentos de la sociedad. Hay poblaciones que necesitan atención especial por su completa dependencia del ambiente que otros cuidan, como los confinados en reformatorios o instituciones psiquiátricas, los ancianos o incapacitados que viven solos o en instituciones, los turistas, y los extranjeros en misiones filantrópicas. También hay miles de personas jóvenes y saludables expuestas a la picada de mosquitos mientras están fuera del hogar (por ejemplo, los niños en cuidado preescolar, los estudiantes, los trabajadores y los soldados, entre otros), por lo que la eliminación de los criaderos debe ser una actividad doméstica, gubernamental y empresarial. Cada una de estas situaciones exige una estrategia preventiva diferente; no obstante, la protección de los turistas y de las fuentes de empleo 
en esa industria sigue un esquema, como se verá más adelante, aplicable a otras poblaciones.

El turismo es una de las industrias principales en los países donde el dengue es endémico. En contraposición, la mayoría de los turistas vienen de países donde no saben qué es el dengue, pero se enteran rápidamente si hay una epidemia en el lugar al que se proponen viajar. Así sucedió cuando se desató la epidemia del síndrome respiratorio agudo grave (SARS) en 2003, que redujo considerablemente el flujo de visitantes a las ciudades afectadas. Según una revista de negocios, la epidemia de 1994 fue uno de los principales acontecimientos adversos para la economía de Puerto Rico en la década de 1987 a 1996 (33). Tanto los turistas como las empresas turísticas reaccionan con gran ansiedad ante las noticias de epidemia de dengue.

Asegurarle al posible visitante, sin más detalles, que no hay peligro para su salud solo estimula su escepticismo. En 1994 y 1998, las llamadas de personas que deseaban viajar a Puerto Rico se canalizaron a través de oficiales de salud pública que ofrecían orientaciones acerca del riesgo de enfermedad según las circunstancias particulares de cada viaje. En 1994, el gobierno recurrió a una compañía de relaciones públicas de Nueva York para que elaborara materiales informativos que ayudaran a los agentes de viajes a orientar a los posibles viajeros. Esta agencia consultó a los Centros para el Control y la Prevención de Enfermedades (CDC) de los Estados Unidos para hacer más comprensibles los datos básicos referentes a la transmisión del dengue. Una alianza intersectorial de los CDC, el Departamento de Salud, la Compañía de Turismo (gubernamental) y la Asociación de Hoteles de Puerto Rico permitió desarrollar una estrategia para la orientación y la inspección de las hostelerías durante las epidemias, proveerles material educativo y entrenar a su personal para que inspeccionara la planta física y eliminara los criaderos que pudiera haber. Luego, el Departamento de Salud hacía una inspección y, si era satisfactoria, expedía un certificado de aprobación. De esta forma, los hoteles podían contestar muchas de las preguntas de sus clientes y demostrar que actuaban para garantizar la salud de sus huéspedes.

\section{La atención a los pacientes}

La carga principal que impone el dengue a los países afectados no radica en el número de muertes, sino en el enorme número de hospitalizaciones y de días de enfermedad (34). El cuidado eficaz de los pacientes durante las epidemias es cualitativamente diferente del que se necesita para la atención individual. El cuidado de un elevado número de casos de dengue exige médicos y personal de enfermería entrenados, criterios de triaje rápido, camas y materiales, así como equipo y guías de tratamiento y aislamiento. El aislamiento no solo se refiere a las precauciones habituales para la manipulación de la sangre y de otros líquidos corporales, sino también al uso de mosquiteros. Si en el hospital hay mosquitos Aedes se debe evitar que piquen a los pacientes con viremia (febriles) y transmitan la infección en la comunidad.

Las guías redactadas por expertos convocados por la OMS, la OPS y otras organizaciones regionales y nacionales brindan recomendaciones similares, basadas en la experiencia del hospital infantil Queen Sirikit National Institute of Child Health, de Bangkok, Tailandia, aunque su implantación puede seguir esquemas radicalmente diferentes (10-12, 35-37). El éxito de ese modelo se basa en el entrenamiento de los profesionales para la captación temprana de los casos y en la educación de la comunidad para que acuda al médico ante la presencia de síntomas de dengue.

Las recomendaciones elaboradas por la OMS en 1986 para planificar suministros suficientes durante una epidemia de dengue hemorrágico se basaron en la tasa de hospitalización registrada durante la epidemia de dengue en Cuba ( $1 \%$ de la población) (38). La experiencia posterior ha permitido funcionar con niveles de ingreso hospitalario mucho menores. Durante la epidemia más grave ocurrida en Puerto Rico (1994-1995), el sistema de notificación registró 1,3 hospitalizaciones por sospecha de dengue por cada 1000 habitantes. Los hospitales notificaron una mediana de $3 \%$ de sus camas ocupadas por casos de dengue confirmados o sospechados durante los tres meses centrales (pico) de la epidemia (intervalo: 0,2\% a 47,0\%; la mitad de los hospitales notificaron de $1 \%$ a $6 \%$ ) (17). Se debe aclarar que en Puerto Rico hay cerca de 60 hospitales - casi todos privados-con condiciones adecuadas para atender a los pacientes con dengue mientras su estado no sea de extrema gravedad.

Para prevenir las muertes se deben emprender actividades que vayan más allá del portón de las instituciones clínicas, como la educación de la población para que no tome aspirinas, mantenga al paciente bien hidratado por vía oral y reconozca las señales de peligro. También se necesita un buen sistema de transporte de urgencia para que el paciente llegue vivo al centro que lo puede atender adecuadamente. En el Hospital Pediátrico No. 1 de Ciudad Ho Chi Minh, Vietnam, los criterios establecidos para el traslado selectivo de casos de dengue con pronóstico grave son las hemorragias masivas (que pueden necesitar transfusiones), la encefalopatía, la insuficiencia respiratoria o hepática, el choque re- 
currente o prolongado, o la presencia de otra enfermedad subyacente (Dr. Nguyen Thanh Hung, 2001, comunicación personal). Antes del traslado es necesario intentar sacar al paciente del estado de choque, avisar con antelación al hospital al que se le refiere, acompañarlo de un documento de referencia escrito con claridad y cerciorarse de que la ambulancia cuente con un sistema de administración de oxígeno y un enfermero o médico que viaje con el paciente.

\section{Educación médica}

Los puntos esenciales de la educación médica se remiten a las complejidades del desarrollo de la enfermedad y a su tratamiento. En la página de Internet de los CDC se pueden encontrar diapositivas para la educación sobre el dengue en inglés, portugués y español (http://www.cdc.gov/ncidod/ dvbid/dengue/slideset/spanish/). Se debe subrayar que el tratamiento del dengue consiste en tomar acetaminofén para aliviar el dolor y la fiebre (nunca aspirina o fármacos antiinflamatorios no esteroideos), y abundantes líquidos. Por lo general, los profesionales clínicos solo conocen la definición textual del mosquito Ae. aegypti ("con bandas blancas en las patas"), por lo que vale la pena enseñarles frascos con las distintas etapas del ciclo de vida del mosquito (huevos, larvas, pupas y mosquitos adultos) para que conozcan su tamaño real, aprendan a reconocer las larvas y puedan transmitir esos conocimientos a sus pacientes. Además, se debe insistir en que, en el momento del contacto médico, el paciente reciba orientación sobre cómo eliminar los mosquitos adultos y los criaderos de mosquitos de su casa y los alrededores y sobre cómo evitar las picadas.

La educación médica sobre el dengue hemorrágico enfrenta varios obstáculos, entre ellos el problema semántico, pues el propio nombre parece indicar que las hemorragias son el mayor riesgo para la vida del paciente y que si se sangra, "es por dengue hemorrágico". Ambos errores son peligrosos, ya que el primero desvía la atención del médico de los verdaderos signos de alarma y el segundo conduce a hospitalizaciones innecesarias y al hacinamiento en los hospitales. La definición de un caso de dengue hemorrágico abarca cuatro criterios: fiebre, trombocitopenia, cualquier manifestación hemorrágica e hiperpermeabilidad vascular. Aunque los cuatro criterios deben estar presentes, el último síntoma es el más peligroso, pues es el que ocasiona la hipotensión y el choque por dengue. La documentación de estos cuatro criterios exige una observación atenta y pruebas de laboratorio repetidas mientras dure la enfermedad.
FIGURA 2. Señales de alerta del choque por dengue

Choque por dengue

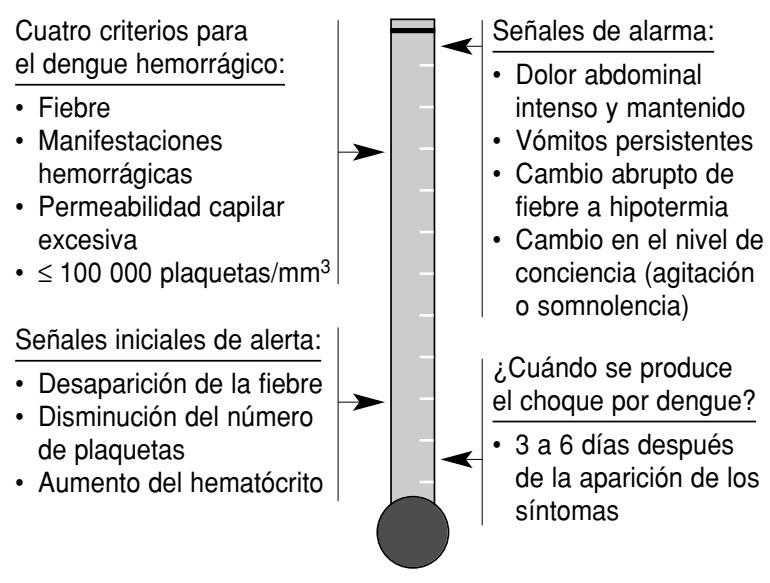

La prevención del choque por dengue requiere de un tratamiento precoz, antes de que la hipotensión sea evidente. Para evitar esto, en Costa Rica se estableció que la atención del paciente con dengue tiene como uno de sus objetivos mantener la presión arterial media en $70 \mathrm{~mm} \mathrm{Hg}$ o más en los adultos (en los niños, se debe mantener el valor apropiado para la edad) y una excreción urinaria superior a $1 \mathrm{~mL} / \mathrm{kg}$ de peso por hora (39).

Para ayudar al personal médico a recordar estos conceptos y darle una orientación dinámica acerca del cuidado de los casos, en Puerto Rico se elaboró la gráfica del "termómetro" (figura 2). Esta refleja de manera cualitativa el riesgo creciente de choque y muerte por dengue según el estadio de la enfermedad en el que se encuentre el paciente en el momento de la evaluación, es decir, el tiempo transcurrido desde el comienzo de los síntomas, la documentación de los criterios de dengue hemorrágico y las señales de alarma (40-42). Para hacer mayor hincapié en las formas poco frecuentes y más graves de dengue se presenta también un esquema con las "rutas a la muerte por dengue" (figura 3), que señala los cinco síndromes causados por el virus del dengue que pueden llevar a la muerte: el choque por extravasación de líquidos, la insuficiencia hepática, la miocardiopatía, la encefalopatía y la hemorragia gastrointestinal masiva abrupta (sin desarrollar las otras etapas del dengue hemorrágico). Por lo general, los médicos y la población se angustian por la posibilidad de una hemorragia y están muy atentos a las plaquetas (el camino estrecho y poco transitado, con letreros y policías), pero olvidan que la más frecuente de todas las manifestaciones graves de dengue (la carretera congestionada) es el cho- 
FIGURA 3. Las rutas a la muerte por dengue

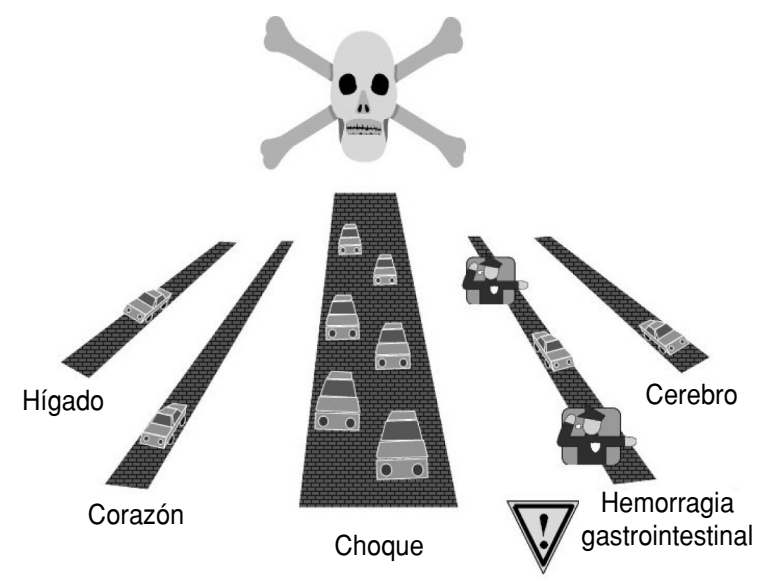

que, que puede ser súbito, y relegan la vigilancia de la presión arterial a mediciones rutinarias cada cuatro u ocho horas. El médico que solamente se preocupa por prevenir las hemorragias no está cuidando adecuadamente del paciente de dengue.

Aún falta información para poder elaborar un esquema indicativo de las señales de mejoría o de fin de la crisis. ¿Cuán alentador es el "signo de la arepa", es decir, el regreso del apetito expresado por el niño venezolano al pedir una arepa (Iris Villalobos de Chacón, Maracay, Venezuela, comunicación personal)? ¿Cuán indicativos de que el paciente salió del período crítico pueden ser la aparición del prurito, la diuresis, la bradicardia sinusal o los linfocitos atípicos, aunque todavía haya una trombocitopenia grave? Aún no se sabe con certeza.

\section{La investigación}

Durante una epidemia, todas las muertes con hemorragia se atribuyen al dengue. Es importante lograr que el médico sepa diferenciar entre los cuadros típicos y atípicos de dengue debido a la posible importación de la fiebre amarilla, ya que el vector es el mismo para ambas enfermedades. Para confirmar el diagnóstico de un caso importante - por tener síntomas poco frecuentes, un desenlace mortal o indicios de otra enfermedad- es indispensable contar con el respaldo del laboratorio de diagnóstico y de patólogos entrenados en obtener y analizar muestras de los tejidos afectados (43). Incluso si la localidad o el país no cuentan con esos recursos, hay laboratorios nacionales y regionales en nuestra área capacitados y dispuestos a servir como refuerzo en estas situaciones. Lo importante es saber cómo utilizarlos cuando se presenta una emergencia.

Las epidemias se caracterizan por el aumento del número de enfermos, acompañado por el aumento de la proporción de pacientes cuya dolencia se debe a un agente en particular (en términos epidemiológicos, hay una alta fracción atribuible en la población). Por ello, las epidemias ofrecen el momento más adecuado para identificar las causas y los factores de riesgo de las enfermedades y no se debe desperdiciar esta oportunidad para llevar a cabo una investigación. En última instancia, aun cuando solo se haga "lo mejor posible dentro de las limitaciones", la reflexión minuciosa sobre lo acontecido en una epidemia puede revelar una manera más eficiente o fructífera de ayudar a los pacientes, a sus familias o al personal de salud dedicado a combatir el brote.

\section{La transparencia ante los medios de información}

La transparencia ante los medios de información no se limita solo a la necesidad imperativa de honradez o de gobierno democrático, sino también a la necesidad de educar con claridad y rapidez a la población para que tome las medidas adecuadas, y a los periodistas para que entiendan los detalles de un problema complejo. Es recomendable elaborar de antemano un comunicado de prensa con información sobre el dengue y su historia local. Las fotos o videos de mosquitos y larvas deben tener una referencia de sus dimensiones (por ejemplo, una moneda pequeña o la punta de un lápiz, mejor que una escala métrica) para que la población entienda mejor el tamaño real de los organismos.

La curiosidad hace que los ciudadanos se fijen en el número de casos y en los detalles de los que tienen un desenlace fatal. Los informes oficiales deben hacer hincapié en las tendencias que muestra la incidencia (alta, baja, circunscrita) y no solo en las cifras específicas. Es recomendable identificar un solo portavoz para la información oficial, ya que sin faltar a la verdad, el laboratorio, el hospital y la oficina de vigilancia epidemiológica podrían cada uno recibir diferente número de casos de dengue en una misma semana.

Este portavoz debe ser capaz de responder prontamente a las preguntas que le formulen, debe describir los casos sin dramatismo, respetando el derecho de los pacientes a la privacidad, y debe evitar hacer pronósticos acerca de la brevedad de la epidemia, ya que estos pueden hacer disminuir prematuramente las medidas de control y, en caso de no cumplirse ese pronóstico, restarle credibilidad a 
la acción del gobierno. Toda comunicación dirigida a la población debe indicar, además, qué puede hacer cada ciudadano ante la situación del momento (medidas de protección personal, del ambiente, del enfermo, etc.). La repetición de datos básicos sobre la prevención es siempre útil, pues cada noticia atrae a nuevos lectores que antes no consideraban el asunto importante.

\section{CONCLUSIONES}

¿Cómo responder a una epidemia de dengue? Con una buena preparación dirigida a cumplir la meta fundamental: prevenir la infección y minimizar sus efectos en las personas enfermas. La mejor forma de responder a ese "huracán viral" que constituye cada epidemia de dengue es prever su aparición. La improvisación puede indicar flexibilidad y originalidad, pero confiar a la improvisación la solución de todos los problemas que se presentan en momentos de crisis refleja una actitud ineficiente, temeraria e irresponsable. Como ocurre con los huracanes, cuando llegan la lluvia y los vientos, las personas no deben andar en busca de abrigo, sino estar ya cobijadas y alertas para atender a las consecuencias imprevistas de las fuerzas de la naturaleza.

\section{SYNOPSIS}

\section{How to respond to a dengue epidemic: overview and experience in Puerto Rico}

Dengue, a viral disease transmitted by mosquitoes, is endemic and frequently epidemic in many tropical countries. Because low-incidence periods vary in length, it is difficult to know in advance when an epidemic will occur. Response efforts, despite being logical, have been counter-productive at times. Furthermore, experience has demonstrated that dengue epidemics last a long time, making it important that government control efforts be sustainable while they last.

This article describes priority activities requiring attention in order to minimize the impact of dengue epidemics. Such activities, which in many cases can be adapted to combat other types of epidemics as well, are as follows: (1) establishment of an inter-sectoral action committee, (2) formalization of an emergency action plan, (3) epidemiologic surveillance, (4) diagnostic laboratory testing, (5) mosquito control, (6) protection of sources of employment and special populations, (7) patient care, (8) education of medical personnel, (9) research, and (10) transparency before the mass media.

The best way to reduce the ravaging effects of dengue epidemics is to anticipate their emergence so that infection can be prevented and steps can be taken to protect the ill. Relying on improvisation to solve all the problems that arise in moments of crisis is inefficient and reckless.

Key words: dengue; disease outbreaks; epidemiologic surveillance.

\section{REFERENCIAS}

1. Gubler DJ, Clark GG. Community involvement in the control of Aedes aegypti. Acta Tropica. 1996;61:169-79.

2. Sorprende virulencia del dengue. El Diario de Hoy [San Salvador, El Salvador]. 200024 de agosto:1-3.

3. Furney WJ. Fulmina el dengue a 235 indonesios. El Nuevo Día [San Juan, Puerto Rico]. 200423 de febrero:82.

4. DeRoeck D, Deen J, Clemens JD. Policymakers' views on dengue/dengue haemorrhagic fever and the need for dengue vaccines in four Southeast Asian countries. Vaccine. 2003;22:121-9.

5. Rigau-Pérez JG, Ayala-López A, GarcíaRivera EJ, Hudson SM, Vorndam V, Reiter $\mathrm{P}$, et al. The reappearance of dengue- 3 and subsequent dengue- 4 and dengue-1 epidemics, Puerto Rico, 1998. Am J Trop Med Hyg. 2002;67:355-62.

6. Gubler DJ. Aedes aegypti and Aedes aegypti-borne disease control in the 1990s: top down or bottom up. Am J Trop Med Hyg. 1989;40:571-8.

7. Gubler DJ, Casta-Vélez A. A program for the prevention and control of epidemic dengue and dengue hemorrhagic fever in Puerto Rico and the U.S. Virgin
Islands. Bull Pan Am Health Organ. 1991;25:237-47.

8. Gubler DJ. How effectively is epidemiological surveillance used for dengue programme planning and epidemic response? Dengue Bull. 2002;26:96-106.

9. San Martín JL, Prado M. Percepción del riesgo y estrategias de comunicación social sobre el dengue en las Américas. Rev Panam Salud Publica. 2004;15:135-9.

10. World Health Organization. Dengue haemorrhagic fever: diagnosis, treatment, prevention and control. 2. ${ }^{\mathrm{a}}$ ed. Geneva: WHO; 1997.

11. Organización Panamericana de la Salud. Dengue y dengue hemorrágico en las Américas: guías para su prevención y control. Washington, D.C.: OPS; 1995. (Publicación científica No. 548). Hallado en: http://www.paho.org/common/ Display.asp?Lang=E\&RecID $=4479$. Acceso el 25 de diciembre de 2004.

12. Regional Office for South-East Asia, World Health Organization. Prevention and control of dengue and dengue haemorrhagic fever: comprehensive guidelines. New Delhi: World Health Organization; 1999. (Regional Publica- tion 29/1999). Hallado en: http://w3. whosea.org/en/Section10/Section332/ Section554.htm. Acceso el 24 de diciembre de 2004

13. Organización Panamericana de la Salud. Marco de referencia: nueva generación de programas de prevención y control del dengue en las Américas. Washington, D.C.: OPS; 2002. (OPS/HCP/HCT/ 206/02). Hallado en: http://www.paho. org/Spanish/HCP/HCT/VBD/denguenueva-generacion.htm. Acceso el 24 de diciembre de 2004.

14. Federal Emergency Management Agency. State and local guide 101: guide for allhazard emergency operations planning. Washington, D.C.: FEMA; 1996. Hallado en: http://www.fema. gov/pdf/rrr/ slg101.pdf. Acceso el 25 de diciembre de 2004.

15. Centers for Disease Control and Prevention. Framework for evaluating public health surveillance systems for early detection of outbreaks; recommendations from the CDC Working Group. MMWR. 2004;53(RR-5):1-13.

16. World Health Organization. Dengue fever: outbreak of classic dengue, Costa 
Rica. Weekly Epidemiol Rec. 1994;69: 85-6.

17. Rigau-Pérez JG, Vorndam AV, Clark GG. The dengue and dengue hemorrhagic fever epidemic in Puerto Rico, 1994-1995. Am J Trop Med Hyg. 2001; 64:67-74.

18. Rigau-Pérez JG, Millard PS, Walker DR, Deseda CC, Casta-Vélez A. A deviation bar chart for detecting dengue outbreaks in Puerto Rico. Am J Public Health. 1999; 89:374-8.

19. Heffernan R, Mostashari F, Das D, Karpati A, Kulldorff M, Weiss D. Syndromic surveillance in public health practice, New York City. Emerg Infect Dis. 2004;10:858-64.

20. Kafka F. Parables and paradoxes. New York: Schoken Books; 1958. Hallado en http://www.eigengrau.com/kafka/ poseidon.html. Acceso el 25 de enero de 2005.

21. Kübler-Ross E. On death and dying. New York: Macmillan; 1969

22. Epstein RM, Alper BS, Quill TE. Communicating evidence for participatory decision making. JAMA. 2004;291:2359-66.

23. Rigau-Pérez JG, Gubler DJ. Surveillance for dengue and dengue haemorrhagic fever. En: Gubler DJ, Kuno G, eds. Dengue and dengue haemorrhagic fever. Wallingford, U.K.: CAB International; 1997. Pp. 405-23.

24. Chen LH, Wilson ME. Transmission of dengue without a mosquito vector: nosocomial mucocutaneous transmission and other routes of dengue transmission. Clin Infect Dis. 2004;39:e56-60.

25. Centers for Disease Control and Prevention. Update: West Nile Virus screening of blood donations and transfusionassociated transmission-United States, 2003. MMWR. 2004;53:281-4.

26. Reiter P, Nathan MB. Guidelines for assessing the efficacy of insecticidal space sprays for control of the dengue vector Aedes aegypti. Geneva: World Health Organization; 2001. (WHO/CDS/CPE/ PVC/2001.1).

27. Castle T, Amador M, Rawlins S, Figueroa JP, Reiter P. Absence of impact of aerial malathion treatment on Aedes aegypti during a dengue outbreak in Kingston, Jamaica. Rev Panam Salud Publica. 1999;5:100-5.

28. Morrison AC, Getis A, Santiago $M$ Rigau-Pérez JG, Reiter P. Exploratory space-time analysis of reported dengue cases during an outbreak in Florida Puerto Rico, 1991-1992. Am J Trop Med Hyg. 1998;58:287-98.

29. Palmer CJ, Validum L, Vorndam AV Clark GG, Validum C, Cummings R, et al. Dengue in Guyana. Lancet. 1999; 354:304.

30. Centers for Disease Control and Prevention. Underdiagnosis of dengueLaredo, Texas, 1999. MMWR. 2001;50: 57-9.

31. Hayes JM, García-Rivera EJ, FloresReyna R, Suárez-Rangel G, RodríguezMata T, Coto-Portillo R, et al. Risk factors for infection during a severe dengue outbreak, El Salvador, 2000. Am J Trop Med Hyg. 2003;69:629-33.

32. Renganathan E, Parks W, Lloyd L, Nathan MB, Hosein E, Odugleh A, et al. Towards sustaining behavioral impact in dengue prevention and control. Dengue Bull. 2003;27:6-12.

33. Dimeo S. Tough times for Puerto Rico. Caribbean Business. 1997;25(7):29.

34. Gubler DJ, Meltzer MI. The impact of dengue/dengue hemorrhagic fever on the developing world. En: Maramorosch K, Murphy FA, Shatkin AJ, eds. Advances in virus research. 1999;53:35-70.

35. Yunus EB, Mannan Bangali A, Ataul Huq Mahmood M, Mushfiqur Rahman
M, Chowdhury AR, Talukder KR. Dengue outbreak 2000 in Bangladesh: from speculation to reality and exercises. Dengue Bull. 2001;25:15-20.

36. Lemus Lago ER, Estévez Torres G, Velásquez Acosta JC. Campaña por la esperanza: la lucha contra el dengue. La Habana: Editora Política; 2002.

37. Alfaro A, Pizarro D, Navas L, Kivers G, Penniecook T, Pérez E. La organización y efectividad de una unidad especial de atención de dengue del área de salud de Limón, Costa Rica, 1999. Memorias de la Academia Nacional de Ciencias (Costa Rica). 2001;7(El Dengue):11-21.

38. World Health Organization. Dengue haemorrhagic fever: diagnosis, treatment and control. Geneva: WHO; 1986.

39. Alfaro A, Navas L, Pérez E, Barrantes J, Rodríguez M, Pizarro D, et al. Hallazgos clínicos detectados al ingreso en los pacientes hospitalizados para predecir la evolución a dengue hemorrágico. Memorias de la Academia Nacional de Ciencias (Costa Rica). 2001;7(El Dengue): 49-66.

40. Nimmannitya S. Dengue fever/dengue haemorrhagic fever: case management. Trop Med (Nagasaki). 1994;36:249-56.

41. Martínez Torres E. Dengue y dengue hemorrágico: aspectos clínicos. Salud Publica Mex. 1995;37(Supl):29-44.

42. Méndez A, González G. Dengue hemorrágico en niños: diez años de experiencia clínica. Biomédica. 2003;23:180-93.

43. Gubler DJ, Zaki SR. Dengue and other viral hemorrhagic fevers. En: Nelson AM, Horsburgh CR, Jr., eds. Pathology of emerging infections 2. Washington, D.C.: American Society for Microbiology; 1998. Pp. 43-71.

\section{Dengue y dengue hemorrágico en las Américas: guías para su prevención y control}

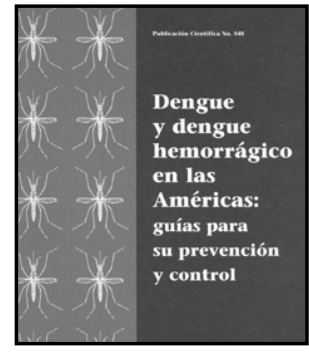

$1995 \cdot 116 \mathrm{pp}$. ISBN 9275315485

Código: PC 548

Precio: US\$25.00/

US\$ 18.00 en América Latina y el Caribe
Las pautas presentadas en esta publicación subrayan el hecho de que el dengue es un problema vinculado principalmente con el saneamiento del medio domiciliario. Los vectores más importantes del dengue, Aedes aegypti y Aedes albopictus, se pueden controlar mediante maniobras físicas y sin el uso excesivo de sustancias químicas. A diferencia de los antiguos programas centralizados y de estructura vertical, estas pautas hacen hincapié en las distintas formas de transferir a la comunidad la responsabilidad, capacidad y motivación que requieren el control y la prevención del dengue.

La presente publicación, que ha sido preparada por 18 representantes de siete países de las Américas y varios funcionarios de la OPS, todos expertos en el tema tratado, ocupa un lugar crítico en la lucha contra el dengue y constituye un instrumento indispensable para profesionales de la salud que participan en el control de vectores.

Usted también puede enviar su pedido y pago en dólares estadounidenses a: http://publications.paho.org Fax: (301) 206-9789• E-mail: paho@pmds.com•Oficina de la OPS/OMS en su país. 